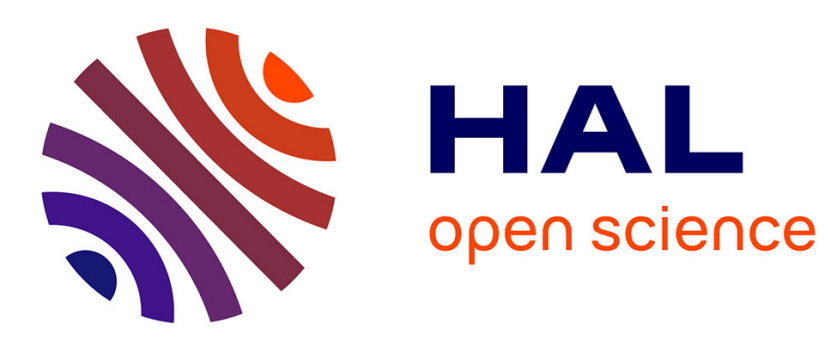

\title{
On systolic zeta functions
}

\author{
Ivan Babenko, Daniel Massart
}

\section{To cite this version:}

Ivan Babenko, Daniel Massart. On systolic zeta functions. European Journal of Mathematics, 2017, 3 (4), pp.899-915. 10.1007/s40879-017-0181-1 . hal-01516391

\section{HAL Id: hal-01516391 \\ https://hal.science/hal-01516391}

Submitted on 30 Apr 2017

HAL is a multi-disciplinary open access archive for the deposit and dissemination of scientific research documents, whether they are published or not. The documents may come from teaching and research institutions in France or abroad, or from public or private research centers.
L'archive ouverte pluridisciplinaire HAL, est destinée au dépôt et à la diffusion de documents scientifiques de niveau recherche, publiés ou non, émanant des établissements d'enseignement et de recherche français ou étrangers, des laboratoires publics ou privés. 


\title{
ON SYSTOLIC ZETA FUNCTIONS
}

\author{
IVAN BABENKO ET DANIEL MASSART
}

\begin{abstract}
We define Dirichlet type series associated with homology length spectra of Riemannian, or Finsler, manifolds, or polyhedra, and investigate some of their analytical properties. As a consequence we obtain an inequality analogous to Gromov's classical intersystolic inequality, but taking the whole homology length spectrum into account rather than just the systole.
\end{abstract}

\section{INTRODUCTION}

Let $(M, g)$ be a $m$-dimensional Riemannian manifold. Let $l$ be the shortest possible length of a closedl geodesic whose homology class is non-trivial in $H_{1}(M, \mathbb{R})$. This length is called homological systole of $(M, g)$ and denoted $l=\operatorname{sys}_{H}(M, g)$. It carries important information about the manifold $(M, g)$. As was discovered by Gromov ([11]), under topological conditions on $\mathrm{M}$ to be explained below, the following inequality holds for all Riemannian metric $\mathrm{g}$ on $\mathrm{M}$ :

$$
\sigma_{m}\left(\operatorname{sys}_{H}(M, g)\right)^{m} \leq \operatorname{vol}(M, g),
$$

where $\sigma_{m}$ is a universal constant only depending on the dimension $m$, whose optimal value is unknown. Now let us explain the topological condition under which Inequality (1) holds. Recall that for any manifold M there is a map, called characteristic map, unique modulo homotopy,

$$
f: M \longrightarrow \mathbb{T}^{b_{1}}
$$

where $b_{1}=b_{1}(M)$ is the first Betti number of $\mathrm{M}$ and $T^{b_{1}}$ is the $b_{1}$-dimensional torus, such that the induced map

$$
f_{*}: H_{1}(M, \mathbb{Z}) / \text { Tors } \longrightarrow H_{1}\left(T^{b_{1}}, \mathbb{Z}\right),
$$

where Tors means the torsion subgroup of $H_{1}(M, \mathbb{Z})$, is an isomorphism of $\mathbb{Z}$-modules.

Let $\alpha[M]$ denote $f_{*}([M]) \in H_{m}\left(M, \mathbb{Z}_{\star}\right)$, where $[M]$ is the fundamental class of $M$ and $\mathbb{Z}_{\star}=\mathbb{Z}$ when $M$ is orientable and $\mathbb{Z}_{2}$ otherwise. A sufficient condition for (1) to hold for any metric $g$ is that $\alpha[M] \neq 0$. It can be shown to be also necessary.

In this paper, instead of just the homological systole, we shall consider what we call the homology length spectrum of $(M, g)$. For any $\theta \in H_{1}(M, \mathbb{Z}) /$ Tors , denote $l_{\theta}$ the smallest possible length of a closed geodesic in the homology class $\theta$. Denote $\Theta=H_{1}(M, \mathbb{Z}) /$ Tors $\backslash$ $\{0\}$ the set of non-trivial homology classes and define the homology length spectrum of $(M, g)$ as $L_{\Theta}=L_{\Theta}(g)=\left\{l_{\theta}\right\}_{\theta \in \Theta}$. Of course $\operatorname{sys}_{H}(M, g)=\inf L_{\Theta}$. The goal of this paper is to extract geometric and topological information from the homology length spectrum as it was done from the systole. The information carried by the homological length spectrum $L_{\Theta}$ is conveniently encoded in the formal series

Partially supported by the grants RFSF 10-01-00257-a, and ANR Finsler. 


$$
\zeta_{\text {sys }}(z)=\sum_{\theta \in \Theta} \frac{1}{l_{\theta}^{z}}
$$

which we call systolic zeta function of $(M, g)$. In Chapter 2 we show that the formal series (3) converges in a natural sense and enjoys interesting analytical properties.

To explain our main results we need to define the stable norm of $(M, g)$. Any metric $g$ on $M$ induces a norm $\|\cdot\|_{s t}^{g}$ on $H_{1}(M, \mathbb{R})$ called stable norm (see [10] and chapter 2 for more detail). Denote by $B_{g}(1)$ the unit ball of the stable norm. Let $V(g)$ be the volume of $B_{g}(1)$ with respect to the Haar measure on $H_{1}(M, \mathbb{R})$, normalised in such a way that the quotient of $H_{1}(M, \mathbb{R})$ by the lattice $H_{1}(M, \mathbb{Z}) /$ Tors has volume 1 .

Theorem 1.1. For any Riemannian manifold $(M, g)$, the series (3) converges for all $z$ such that $\operatorname{Re}(z)>b_{1}(M)$, and diverges when $\operatorname{Re}(z)<b_{1}(M)$. The function $\zeta_{\text {sys }}(z)$ is holomorphic in the half-plane $\operatorname{Re}(z)>b_{1}(M)$.

Furthermore the function $\zeta_{\text {sys }}(z)$ extends analytically to the half-plane $\operatorname{Re}(z)>b_{1}-1$, with a simple pole at $z=b_{1}$ with residue $\operatorname{Res}_{b_{1}}\left(\zeta_{\text {sys }}(z)\right)=b_{1}(M) V(g)$.

This results comes as a particular case of Theorem 5.2, proven in Chapter 2, which holds for any Riemannian or Finsler polyhedron.

The analytical properties of $\zeta_{\text {sys }}(z)$ are reflected in the homology length spectrum, which enables us to prove an inequality analogous to (1). Furthermore our approach allows us to estimate the constants that appear in the inequality.

Theorem 1.2. Let $M$ be a an orientable $m$-dimensional differentiable manifold with first Betti number $b_{1}$. For any Riemannian metric $g$ on $M$ we have the following inequality:

$$
\left(\frac{b_{1} v_{b_{1}} V[M]}{\operatorname{Res}_{b_{1}}\left(\zeta_{\text {sys }}(z)\right)}\right)^{m} \leq(\operatorname{vol}(M, g))^{b_{1}} .
$$

Here $V[M]$ is a topological invariant called algebraic volume, which is defined in Chapter 6. In the particular case $m=b_{1}$ it is just to the degree of the characteristic map : $V[M]=|\operatorname{deg}(f)|$. The number $v_{n}$ is a universal constant which comes from the solution of an optimization problem in $n$-dimensional convex geometry. Although the exact value of $v_{n}$ is unknown when $n \geq 3$, there are explicit estimates which are asymptotically correct when $n$ goes to infinity. See Chapter 6 for more detail and the proof of Theorem 1.2 .

The classical Riemann zeta $\zeta(z)=\sum_{n=1}^{\infty} \frac{1}{n^{z}}$ appears naturally in the previous context like as the systolic zeta of function of the simplest manifold, the circle $S^{1}$. In this case for a metric $g$ of unit volume (or length) we have

$$
\zeta_{\text {sys }}(z)=2 \zeta(z)
$$

Another well known number theoretic zeta function is the so called Hurwitz zeta function

$$
\zeta(z ; q)=\sum_{n=0}^{\infty} \frac{1}{(q+n)^{z}},
$$

where $q, \quad R e q>0$ is a complex parameter. See [1] for details and analytic properties as well as connections of Hurwitz zeta functions for rational parameter $q$ with Dirichlet $L$-functions. 
Hurwitz zeta functions appear naturally in the systolic setting as well. More precisely in chap. 3 we define the stable systolic zeta functions for Riemannian polyhedra. Weighted graphs are the simplest example. A graph is called combinatorial if the weight of each edge is equal to 1 .

In chapter 3 the stable systolic zeta function for a combinatorial graph is explicitly calculated as a linear combination of Hurwitz zeta functions, see Theorem 3.2 for a precise statement. Such a representation of the stable systolic zeta function for a graph provides an analytic extension of the stable systolic zeta function to the whole complex plane as a meromorphic function, see chap. 3 for details.

All results in this paper are presented in the more familiar Riemannien context. Nevertheless, up to Chapter 6 all of them can be directely translated to the Finsler setting. Moreower a stable systolic zeta can be seen as a Finsler zeta on the corresponding flat finsler torus. To be expressed in the Finsler language, the results of Chapter 6 would require more precise statements and a suitable choice of a Finsler volume.

\section{Systolic Zeta FunCtion}

Let us consider a countable set $\Theta$ and a map $l: \Theta \longrightarrow \mathbb{R}_{+}$. Let

$$
L_{\Theta}=\left\{l_{\theta}\right\}_{\theta \in \Theta}
$$

(or $L$ for short) be the graph of this map, seen as a family of positive numbers, not necessarily pairwise disjoint, indexed by $\Theta$. We say that $L_{\Theta}$ is a $\Theta$-marked spectrum. If we order $L_{\Theta}$ in a natural way, taking multiplicities into account, we get the ordered spectrum

$$
\left|L_{\Theta}\right|=\left\{\left(l_{i}, a_{i}\right)\right\}_{i=1}^{\infty}, \quad l_{1}<l_{2}<\ldots,
$$

where the $a_{i} \in \mathbb{N}$ are the respective multiplicities of the elements $l_{i}, i \in \mathbb{N}$. Let us call zeta function of the family $L$ the formal series

$$
\zeta_{L}(z)=\sum_{\theta \in \Theta} \frac{1}{l_{\theta}^{z}} .
$$

Denote

$$
\Theta_{\leq t}=\left\{\theta \in \Theta \mid l_{\theta} \leq t\right\} \text { and } \Theta_{>t}=\Theta \backslash \Theta_{\leq t} .
$$

Let us say that the series (5) converges for some $z \in \mathbb{C}$ if the sum

$$
\sum_{\theta \in \Theta_{\leq t}} \frac{1}{l_{\theta}^{z}}
$$

is finite for all $t>0$ and has a limit when $t \longrightarrow \infty$. Observe this notion of cenvergence coincides with the greedy convergence introduced in [21], and that the convergence of the series (5) is equivalent to the convergence of the classical Dirichlet series

$$
\zeta_{|L|}(z)=\sum_{i=1}^{\infty} \frac{a_{i}}{l_{i}^{z}},
$$

where $\left(l_{i}, a_{i}\right)$ are the elements of the marked spectrum $\left|L_{\Theta}\right|$. This explains several analytical properties of the function (5). It is easily seen that if the cardinality of $\Theta_{\leq t}$ satisfies

$$
\left|\Theta_{\leq t}\right|=\mathcal{O}\left(t^{b}\right)
$$


then the series (5) converges in the half-plane $R e(z)>b$ and the function $\zeta_{L}(z)$ is holomorphic therein.

Example 2.1. Let us consider a finite simplicial polyhedron $P$ and set

$$
\Theta=\left\{\left(H_{1}(P, \mathbb{Z}) / \text { Tors }\right) \backslash\{0\}\right\} .
$$

Let $g$ be a polyhedral Riemannian metric on $P$ (see e.g. [4]), and for any $\theta \in \Theta$ let us define $l_{\theta}$ as the shortest possible length of a closed geodesic in the homology class $\theta$. The family $L(P, g)=L_{\Theta}$ is well-defined and represents the homology length spectrum of $(P, g)$. Denote $\zeta_{\mathrm{sys}(P, g)}(z)$ (or $\zeta_{\mathrm{sys}}(z)$ for the sake of brevity) the series corresponding (5) for $L_{\Theta}$.

Definition 2.2. The function $\zeta_{\text {sys }}(z)$ defined by (5) for the family $L(P, g)$ is called systolic zeta function of the Riemannian polyhedron $(P, g)$.

\section{Stable Zeta FunCtion}

Let $\mathcal{B}=\left(\mathbb{R}^{b},\|\cdot\|\right)$ be a Banach space, and let $\Gamma \subset \mathbb{R}^{b}$ be a lattice. Let us normalise the Haar measure on $\mathbb{R}^{b}$ so $\Gamma$ has volume 1 . Set $\Theta=\Gamma \backslash\{0\}$ et $L_{\Theta}=\left\{l_{\theta}=\|\theta\|, \theta \in \Theta\right\}$, then $\Theta_{\leq t}=B(t) \cap \Theta$ where $B(t)$ is the ball of radius $t$ centered at 0 in $\mathcal{B}$. Denote $\zeta_{\mathcal{B}}(z)$ the corresponding zeta function.

For instance if $\mathcal{B}_{\nu}=\left(\mathbb{R}^{2},\|\cdot\|_{\nu}\right)$ with $\nu=\{1, \infty\}$, then $\zeta_{\mathcal{B}_{\infty}}(z)=2 \zeta_{\mathcal{B}_{1}}(z)=8 \zeta(z-1)$ where $\zeta$ is Riemann's zeta. In higher dimensions $\zeta_{\mathcal{B}_{\nu}}(z)$, for $\nu=\{1, \infty\}$, may also be expressed in terms of $\zeta$ but we shall not dwell on this topic. In general $\zeta_{\mathcal{B}}(z)$ enjoys the following properties.

Proposition 3.1. Let $\mathcal{B}=\left(\mathbb{R}^{b},\|\cdot\|\right)$ be a Banach space, $\Gamma \subset \mathbb{R}^{b}$ be a lattice, and $V=$ $\operatorname{Vol}(B(1))$ be the volume of the unit ball of $\mathcal{B}$. Then the function $\zeta_{\mathcal{B}}(z)$ satisfies

1) the series (5) converges for all $z$ such that $\operatorname{Re}(z)>b$ and diverges if $\operatorname{Re}(z)<b$.

2) the function $\zeta_{\mathcal{B}}(z)$ extends holomorphically to the half-plane $\operatorname{Re}(z)>b-1$, with $a$ simple pole at $z=b$, with residue $\operatorname{Res}_{b}\left(\zeta_{\mathcal{B}}(z)\right)=b V$.

Proof. Consider the following integer sequence

$$
\{a(n)=|(B(n) \backslash B(n-1)) \cap \Gamma|\}_{n=1}^{\infty}
$$

and set $F(z)=\sum_{n=1}^{\infty} \frac{a(n)}{n^{z}}$. It is easily seen that the series $\zeta_{\mathcal{B}}(z)$ et $F(z)$ convverge in the same half-plane. Since

$$
A(t)=\sum_{n \leq t} a(n)=|B(t) \cap \Gamma|=V t^{b}+\mathcal{O}\left(t^{b-1}\right),
$$

the common half-plane of convergence is $\{z \mid \operatorname{Re}(z)>b\}$ and $z=b$ is singular for both functions. This entails 1 ).

Moreover we have

$$
\zeta_{\mathcal{B}}(z)-F(z)=\sum_{n=1}^{\infty}\left(\sum_{\theta \in(B(n) \backslash B(n-1)) \cap \Gamma}\left(\frac{1}{\|\theta\|^{z}}-\frac{1}{n^{z}}\right)\right) .
$$


Applying, for $z \in \mathbb{R}$, the Mean Value Theorem to the function

$$
\begin{aligned}
f_{z}: \mathbb{R}_{+}^{*} & \longrightarrow \mathbb{C} \\
t & \longmapsto t^{-z}
\end{aligned}
$$

we get that, for all $t$, there exists $h(t) \in] t, E(t)+1[$, where $E($.$) is the floor function,$ such that

$$
f_{z}(E(t))-f_{z}(t)=f_{z}^{\prime}(h(t))(1-\{t\})
$$

where $\{x\}$ is the fractional part of $x$.

Then we have, for $z \in \mathbb{R}$,

$$
\zeta_{\mathcal{B}}(z)-F(z)=z \sum_{n=1}^{\infty}\left(\sum_{n-1<\|\theta\|<n,} \frac{1-\{\|\theta\|\}}{h(\theta)^{z+1}}\right),
$$

with $\|\theta\|<h(\theta)<[\|\theta\|]+1$. Therefore the series (9) converges if $z>b-1$, thus it is holomorphic in the half-plane $\operatorname{Re}(z)>b-1$.

Taking (8) into account, it remains to apply Tauber's Theorem (voir par ex. [22], théorème $2, \S 7.1$ ) to the function $F(z)$. It follows that $F(z)$ extends to $\operatorname{Re}(z)>b-1$ and

$$
F(z)=\frac{b V+o(1)}{z-b}, \text { si } z \longrightarrow b
$$

This ends the proof.

If $(P, g)$ is a Riemannian polyhedron, let us denote $\Gamma=H_{1}(P, \mathbb{Z}) /$ Tors, then $\Gamma$ embeds canonically into $H_{1}(P, \mathbb{R})$. Denote $\Theta(P)=\Gamma \backslash\{0\}$. It is well-known that for all $\theta \in \Gamma$ the following limit

$$
\lim _{n \rightarrow \infty} \frac{l_{n \theta}}{n}
$$

exists and defines a norm on $\Gamma$ which extends by homogeneity and continuity to $H_{1}(P, \mathbb{R})$. This norm is called mass or stable norm, we denote it $\|\cdot\|_{s t}^{g}$ (or $\|\cdot\|_{s t}$ for the sake of brevity). See [10] for several equivalent definitions.

Applying the contruction at the beginning of this section to the Banach space $\mathcal{B}_{s t}=$ $\left(H_{1}(P, \mathbb{R}),\|\cdot\|_{s t}^{g}\right)$ we get the function

$$
\zeta_{s t}(z)=\sum_{\theta \in \Theta(P)} \frac{1}{\|\theta\|_{s t}^{z}}
$$

which we call stable zeta of the Riemannian polyhedron $(P, g)$. Thus it verifies Proposition 3.1 .

Weighted graphs are interesting examples of Riemmannian polyhedra of dimension 1. Such a graph is given by a set of vertices $V$, a set of edges $E$ (we allow loops and multiple edges between two given vertices, and edges are unoriented), and a weight function $w$ : $E \longrightarrow \mathbb{R}_{+}$. We denote $P=(V, E)$, so the weighted graph is $(P, w)$. Each edge is then endowed with a Riemannian metric such that the length of an edge $e$ is $w(e)$. Then the weighted graph $(P, w)$ becomes a dimension 1 Riemannian polyhedron. We say that $(P, w)$ is a combinatorial graph if $w(e)=1$ for all $e \in E$. The only topological invariant of $P$ is its first Betti number $b=b_{1}(P)=|E|-|V|+1$.

The stable norm on $H_{1}(P, \mathbb{R})$ only depends on the weight function $w$ on $P$ and not on the particular choice of the Riemannian metric on each edge. Its unit ball $B_{s t}^{w}$ is always a 
$b$-dimensional polytope whose vertices are in one-to-one correspondance with the simple cycles in $P$, and their number is bounded above by $2\left(2^{b}-1\right)$, see [5] for more detail.

In particular, for any weighted graph $(P, w)$ the stable zeta function $\zeta_{s t}^{w}(z)$ verifies Proposition 3.1. For a general weight function $w$ we cannot expect additional analytical properties for $\zeta_{s t}^{w}(z)$. However our next theorem shows that $\zeta_{s t}^{w}(z)$ does have interesting analytical properties when $(P, w)$ is a combinatorial graph. In the statement $\zeta(s)$ means, as usual, Riemann's zeta.

Theorem 3.2. Let $P$ be a combinatorial graph with first Betti number $b$, and let $V=$ $\operatorname{Vol}\left(B_{\text {st }}\right)$ be the volume of the unit ball of the stable norm in $H_{1}(P, \mathbb{R})$. Then the stable zeta function $\zeta_{s t}(z)$ of the graph $P$ satisfies

1) the series (11) converges for all zsuch that $\operatorname{Re}(z)>b$ and diverges if $\operatorname{Re}(z)<b$.

2) the function $\zeta_{s t}(z)$ extends analytically to the whole complex plane $\mathbb{C}$ as a meromorphic function with simple poles at $z=1,2, \ldots, b$, whose residue at $z=b$ is $\operatorname{Res}_{b}\left(\zeta_{s t}(z)\right)=b V$.

3) there exists $m=m(P) \in \mathbb{N}$ such that

$$
\zeta_{s t}(z)=b V \zeta(z-b+1)+\sum_{l=0}^{b-1} m^{l-z}\left(\sum_{k=1}^{m} p_{l k} \zeta\left(z-l ; \frac{k}{m}\right)\right),
$$

where $\left\{p_{l k}\right\} \in \mathbb{Q}$ and $\zeta(s ; q)$ is the Hurwitz zeta defined in (4).

Proof. Part 1) follows directly from Proposition 3.1. We prove 3) before 2). If $P$ is a combinatorial graph, for all $\theta \in \Theta(P),\|\theta\|_{s t} \in \mathbb{N}$ (see [5]), so we have

$$
\zeta_{s t}(z)=\sum_{\theta \in \Theta} \frac{1}{\|\theta\|_{s t}^{z}}=\sum_{n=1}^{\infty} \frac{A_{n}}{n^{z}}
$$

where $A_{n}$ is the number of points in $\Theta$ with norm $n$. If $B_{s t}(t)$ is the ball of radius $t$, since the norm of any $\theta \in \Theta(P)$ is an integer, we get

$$
A_{n}=\left|B_{s t}(n) \cap \Theta(P)\right|-\left|B_{s t}(n-1) \cap \Theta(P)\right| .
$$

For any combinatorial graph the unit ball $B_{s t}$ of the stable norm is a $b$-dimensional polytope whose vertices are rational with respect to the lattice $H_{1}(P, \mathbb{Z})$ (see [5]). Applying Ehrhart's theorem ([8]), taking the equality $B_{s t}(t)=t B_{s t}$ into account, we get a natural number $m$, which depends only on $B_{s t}$, such that

$$
\left|B_{s t}(n) \cap \Theta(P)\right|=V n^{b}+q(n),
$$

where $q(n)$ is a $m$-quasipolynomial of degree at most $b-1$ with rational coefficients, that is, $q(n)=\sum_{l=0}^{b-1} q_{l}(n) n^{l}$, where $q_{l}(n)$ are $m$-periodic functions. From this and (14) we deduce

$$
A_{n}=b V n^{b-1}+p(n)
$$

where $p(n)=\sum_{l=0}^{b-1} p_{l}(n) n^{l}$ is a $m$-quasipolynomial of degree at most $b-1$ with rational coefficients. Observe that the leading coefficient $p_{b-1}(n)$ averages to zero : $\sum_{k=1}^{m} p_{b-1}(k)=0$. 
This is because $p(n)=q(n)-q(n-1)$ so, setting $q(n)=\sum_{l=0}^{b-1} q_{l}(n) n^{l}$, we get

$$
p(n)=q(n)-q(n-1)=\sum_{l=0}^{b-1} q_{l}(n) n^{l}-\sum_{l=0}^{b-1} q_{l}(n-1)(n-1)^{l}
$$

whose leading coefficient is $q_{b-1}(n)-q_{b-1}(n-1)$, which averages to zero. We shall use this fact in the proof of part 2).

Now we get from (13)

$$
\zeta_{s t}(z)=\sum_{n=1}^{\infty} \frac{b V n^{b-1}+p(n)}{n^{z}}=b V \zeta(z-b+1)+\sum_{l=0}^{b-1}\left(\sum_{n=1}^{\infty} \frac{p_{l}(n)}{n^{z-l}}\right), \operatorname{Re} z>b .
$$

Define a basis $\left\{\alpha_{k}\right\}_{k=1}^{m}$ for the space of $m$-periodic functions on $\mathbb{N}$, by the formula

$$
\alpha_{k}(n)=1 \text { if } n \equiv k \bmod m \text { and } 0 \text { otherwise, for } k=1,2, \ldots, m \text {. }
$$

Set $p_{l}(n)=\sum_{k=1}^{m} p_{l k} \alpha_{k}(n)$, with $p_{l k} \in Q$. From (15) it follows that

$$
\zeta_{s t}(z)-b V \zeta(z-b+1)=\sum_{l=0}^{b-1}\left(\sum_{k=1}^{m} p_{l k} \sum_{n=1}^{\infty} \frac{\alpha_{k}(n)}{n^{z-l}}\right)=\sum_{l=0}^{b-1} m^{l-z}\left(\sum_{k=1}^{m} p_{l k} \zeta\left(z-l ; \frac{k}{m}\right)\right)
$$

which entails (12), thus proving part 3) of the theorem. Part 2) now follows from (12), and the fact that the right-hand side in (12) doesn't have a pole at $z=b$, because $\sum_{k=1}^{m} p_{b-1}(k)=0$.

Remark 3.3. If i $k<m$ are mutually prime integers, it is well known (see [1], Chapter 12) that Hurwitz' zeta function $\zeta\left(s, \frac{k}{m}\right)$ may be expressed as a linear combination of Dirichlet's L-functions. Thus Formula (12) may be rephrased in terms of Dirichlet's L-functions.

\section{Analytical properties of systolic Zeta functions}

For any finite Riemannian polyhedron $(P, g)$ the systolic zeta function $\zeta_{\text {sys }}(z)$ defined in Section 2 satisfies a property analogous to 3.1. Let $P$ be a finite simplicial polyhedron and set

$$
\Theta(P)=\left\{\left(H_{1}(P, \mathbb{Z}) / \text { Tors }\right) \backslash\{0\}\right\} .
$$

For a Riemannian metric $g$ on $P$ we consider the length spectrum $L_{\Theta}=\left\{l_{\theta}\right\}_{\theta \in \Theta}$ and denote $V(g)=\operatorname{Vol}(B(1))$ the volume of the unit ball of the stable norm in $H_{1}(P, \mathbb{R})$.

Theorem 4.1. For any finite Riemannian polyhedron $(P, g)$ the systolic zeta function $\zeta_{\text {sys }}(z)$ satisfies

1) the series

$$
\zeta_{\text {sys }}(z)=\sum_{\theta \in \Theta} \frac{1}{l_{\theta}^{z}}
$$

converges for all $z$ such that $\operatorname{Re}(z)>b_{1}(P)$ and diverges if $\operatorname{Re}(z)<b_{1}(P)$.

2) the function $\zeta_{\text {sys }}(z)$ extends analytically to the half-plane $\operatorname{Re}(z)>b_{1}-1$, with a simple pole at $z=b_{1}$ with residue $\operatorname{Res}_{b_{1}}\left(\zeta_{\text {sys }}(z)\right)=b_{1} V(g)$. 
The proof uses a pair of tools : Proposition 3.1 and the following lemma originally due to D. Burago [6].

Lemma 4.2. For any finite Riemannian polyhedron $(P, g)$ there exists $C>0$ such that

$$
\left|l_{\theta}-\|\theta\|_{s t}\right| \leq C, \quad \theta \in H_{1}(P, \mathbb{Z}) / \text { Tors } .
$$

The proof given in [6] (Theorem 1) applies to the particular case of the torus $T^{m}$, one of the lemmata (Lemma 1) does not extend to the general case. A proof of the general case is published in [7].

Proof of Theorem 4.1. Set $l_{\theta}=\|\theta\|_{s t}+\alpha(\theta)$, Lemma 4.2 entails

$$
|\alpha(\theta)| \leq C, \quad \theta \in \Theta
$$

from which follows the estimate (6) :

$$
\left|\Theta_{\leq t}\right|=\mathcal{O}\left(t^{b_{1}(P)}\right) .
$$

Therefore the series (5) converges if $\operatorname{Re}(z)>b_{1}(P)$ so $\zeta_{\text {sys }}(z)$ is analytical in the half-plane $\operatorname{Re}(z)>b_{1}(P)$. On the other hand the series $\zeta_{s t}(z)$ diverges if $\operatorname{Re}(z)<b_{1}(P)$, whence $\zeta_{\text {sys }}(z)$ also diverges for $\operatorname{Re}(z)<b_{1}(P)$, which proves 1$)$. Now observe that

$$
\zeta_{s y s}(z)-\zeta_{s t}(z)=\sum_{\theta \in \Theta}\left(\frac{1}{l_{\theta}^{z}}-\frac{1}{\|\theta\|_{s t}^{z}}\right)
$$

so, for $z \in \mathbb{R}$,

$$
\zeta_{s y s}(z)-\zeta_{s t}(z)=-z \sum_{\theta \in \Theta}\left(\frac{\alpha(\theta)}{\left(\|\theta\|_{s t}+\beta(\theta)\right)^{z+1}}\right),
$$

with $|\beta(\theta)|<|\alpha(\theta)| \leq C$. The series (16) converges for $\operatorname{Re}(z)>b_{1}-1$, so Proposition 3.1 implies 2), which ends the proof.

The systolic zeta function $\zeta_{\text {sys }}(z)$ of a Riemannian polyhedron $(P, g)$ encodes a lot of information about $(P, g)$. For instance the ordered homology length spectrum $\left|L_{\Theta}\right|$ may be recovered from $\zeta_{\text {sys }}(z)$. It follows from a classical result of [13] (Theorem 13) that for all $t \notin L_{\Theta}$ and $c>b_{1}(P)$ we have

$$
\left|\Theta_{\leq t}\right|=\frac{1}{2 \pi i} \int_{c-i \infty}^{c+i \infty} \zeta_{s y s}(z) e^{t z} \frac{d z}{z}
$$

The integral (17) is non-decreasing and piecewise constant as a function of $t$. The discontinuities of this function are exactly the points of the ordered length spectrum, and the jumps are the corresponding multiplicities.

On the other hand the metric itself cannot be recovered from $\zeta_{\text {sys }}(z)$. Milnor ([18]) gives an example of two isospectral (therefore having the same systolic zeta function) non-isometric 16-dimensional flat tori, using two lattices in $\mathbb{R}^{16}$ discovered by Witt [23].

In view of formula (17), an important metric invariant of $(P, g)$ is

$$
r(P, g)=\inf \left\{t: \mathbb{Z}\left\langle\Theta_{\leq t}\right\rangle=H_{1}(P, \mathbb{Z}) / \text { Tors }\right\} .
$$

In plain language $r(P, g)$ is the minimal possible length of a basis of the $\mathbb{Z}$-module $H_{1}(P, \mathbb{Z}) /$ Tors. It is known (see [12], Prop. 5.28) that $r(P, g) \leq 2 \operatorname{diam}(P, g)$. 
Example 4.3. Here we consider a closed, orientable Riemannian manifold $(M, g)$ of dimension two. It is known ([15], [16]) that for all $\theta \in \Gamma=H_{1}(M, \mathbb{Z})$ there exist closed geodesics $\gamma_{1}, \ldots \gamma_{k}, k \leq b_{1}(M) / 2$, and integers $\lambda_{1}, \ldots \lambda_{k}$, such that $\theta=\sum_{i=1}^{k} \lambda_{i}\left[\gamma_{i}\right]$ et

$$
\|\theta\|_{s t}=\sum_{i=1}^{k} \lambda_{i}\left\|\left[\gamma_{i}\right]\right\|_{s t}=\sum_{i=1}^{k} \lambda_{i} l_{g}\left(\gamma_{i}\right)
$$

This motivates our interest in the family

$$
\Theta_{\min }:=\left\{\gamma \text { closed geodesic } \mid\|[\gamma]\|_{s t}=l_{g}(\gamma)\right\}
$$

and the corresponding zeta function

$$
\zeta_{\min }(z):=\sum_{\gamma \in \Theta_{\min }} l_{g}(\gamma)^{-z}
$$

From the obvious inclusion

$$
\left\{l_{g}(\gamma) \mid \gamma \in \Theta_{\min }\right\} \subset\left\{\|\theta\|_{s t} \mid \theta \in \Gamma\right\}
$$

follows the convergence of the series $\zeta_{\min }(z)$ in the half-plane $\operatorname{Re}(z)>b_{1}(M)$.

On the other hand, from [17] we know that $\zeta_{\min }(z)$ cannot converge on any half-plane properly containing $\operatorname{Re}(z)>2$. Examples of long-necked surfaces are studied in [17], for such surfaces $\zeta_{\text {min }}(z)$ converges on $\operatorname{Re}(z)>2$, and extends analytically to $\operatorname{Re}(z)>1$.

It would be interesting to determine the domain of convergence of $\zeta_{\min }(z)$. Another question of interest is whether, in the case of a hyperbolic metric $g, \zeta_{\min }(z)$ depends analytically on the metric, thus defining an analytical function on the product of Teichmuller awith a half-plane.

Example 4.4. Let $(P, g)$ be a Riemannian polyhedron and let $\zeta_{\text {sys }}(z)$ be its systolic zeta function. Applying Mellin's transform (see [13], Theorem 11) we get :

$$
\zeta_{\text {sys }}(z)=\sum_{\theta \in \Theta} \frac{1}{l_{\theta}^{z}}=\frac{1}{\Gamma(z)} \int_{0}^{\infty}\left(x^{\frac{z}{2}-1} \sum_{\theta \in \Theta} e^{-l_{\theta}^{2} x}\right) d x,
$$

for $\operatorname{Re}(z)>b_{1}(P)$, where $\Gamma(z)=\int_{0}^{+\infty} t^{z-1} e^{-t} d t$. If $P=T^{b}=R^{n} / \Lambda$ and $g$ is a flat metric, the function

$$
\Theta_{g}\left(\frac{i x}{\pi}\right)=1+\sum_{\theta \in \Theta} e^{-l_{\theta}^{2} x}
$$

it the theta function of the lattice $\Lambda$ (see [20]). For a $b$-dimensional flat torus $\mathbb{R}^{n} / \Lambda$, we obtain the following equality :

$$
\zeta_{s y s}(z)=\zeta_{s t}(z)=\frac{1}{\Gamma(z)} \int_{0}^{\infty} x^{\frac{z}{2}-1}\left(\Theta_{g}\left(\frac{i x}{\pi}\right)-1\right) d x,
$$

where $\operatorname{Re}(z)>b$ and $\boldsymbol{\Theta}_{g}$ is the theta function of the lattice $\Lambda$. The most interesting case is when the lattice is even and unimodular, then $\Theta_{g}$ is a modular form of weight $\frac{b}{2}$, see [20]. 


\section{ThE ZETA MAP}

Let $(M, g)$ be a Riemannian manifold and let $\zeta_{\text {sys }}(z)$ be its systolic zeta function. In this section we investigate some properties of the mapping

$$
Z: g \longrightarrow \zeta_{\text {sys }}(z)
$$

from the set of continuous Riemann metrics on $M$, which we denote $\mathcal{M}(M)$, to the space of holomorphic functions in the half-plane $\left\{\operatorname{Re}(z)>b_{1}(M)\right\}$.

First, let us endow the set $\mathcal{M}(M)$ with a distance function. Let $g_{i} \in \mathcal{M}(M), i=1,2$ be continuous Riemann metrics on $M$, and set

$$
\varrho\left(g_{1}, g_{2}\right)=\sup _{q \in M}\left(\inf \left\{\rho \in \mathbb{R}_{+} \mid e^{-\rho}\left\|v_{q}\right\|_{g_{1}} \leq\left\|v_{q}\right\|_{g_{2}} \leq e^{\rho}\left\|v_{q}\right\|_{g_{1}} ; v_{q} \in T_{q} M\right\}\right) .
$$

It is easily seen that $\varrho$ makes $\mathcal{M}(M)$ a complete metric space. If $\gamma(t), t \in[0,1]$ is a piecewise smooth curve in $M$, and $\rho=\varrho\left(g_{1}, g_{2}\right)$, then the $g_{i}$-lengths of $\gamma$ are related by the inequalities

$$
e^{-\rho} l^{g_{1}}(\gamma(t)) \leq l^{g_{2}}(\gamma(t)) \leq e^{\rho} l^{g_{1}}(\gamma(t))
$$

It follows that the $g_{i}$-homology length spectra are $\varrho$-close :

$$
e^{-\rho} l_{\theta}^{g_{1}} \leq l_{\theta}^{g_{2}} \leq e^{\rho} l_{\theta}^{g_{1}}, \quad \theta \in \Theta(M) .
$$

Denote $\mathcal{H}(b)$ the space of homolorphic functions in the half-plane $\{\operatorname{Re}(z)>b\}$, topologized by uniform convergence on compact sets. The above inequality implies the following

Proposition 5.1. The mapping $Z: \mathcal{M}(M) \longrightarrow \mathcal{H}\left(b_{1}(M)\right)$ defined in (19) is continuous.

Let $\left(\mathbb{R}^{b}, \Gamma\right)$ be a vector space with a lattice. To any Banach space structure $\mathcal{B}=\left(\mathbb{R}^{b},\|\cdot\|\right)$ on $\mathbb{R}^{b}$ we associate, as in Section 3 , the function $\zeta_{\mathcal{B}}(z)$. Fixing $\Gamma$, and varying the norm $\|\cdot\|$, we define as in (19) a map

$$
Z_{B}: \mathcal{B} \longrightarrow \zeta_{\mathcal{B}}(z)
$$

Denote $B=B(b)$ the set of all norms on $\mathbb{R}^{b}$. Any two norms $\mathcal{B}_{i}=\left(\mathbb{R}^{b},\|\cdot\|_{i}\right), \quad i=1,2$ are equivalent, let $c_{12}$ be the minimal constant such that

$$
c_{12}^{-1}\|\mathbf{x}\|_{1} \leq\|\mathbf{x}\|_{2} \leq c_{12}\|\mathbf{x}\|_{1} \quad \mathbf{x} \in \mathbb{R}^{b} .
$$

Then $\rho\left(\mathcal{B}_{1}, \mathcal{B}_{2}\right):=\ln c_{12}$ defines a distance on $B$. The map (21) is easily seen to be continuous.

Now we would like to describe the image $I(b)=\operatorname{im}\left(Z_{B}\right)$ of the map (21). The group $G L(b)$ operates on $B$ by the rule $\|\mathbf{x}\|_{h}=\left\|h^{-1}(\mathbf{x})\right\|, \quad h \in G L(b)$. This action transfers to zeta functions is such a way that $Z_{B}$ is equivariant. The set $Q(b)=B(b) / G L(b)$ of isomorphism classes of Banach structures on $\mathbb{R}^{b}$, called the Banach-Masur space, is compact with respect to its natural metric. The quotient map

$$
\hat{Z}_{B}: Q(b) \longrightarrow I(b) / G L(b)
$$

is onto. We have just proved the following

Theorem 5.2. The group $G L(b)$ operates in a natural way on $I(b)$. The quotient set $I(b) / G L(b)$ is compact. For any $f \in I(b)$ and any $h \in G L(b)$ we have

$$
\operatorname{Res}_{b} f_{h}=(\operatorname{det} h) \operatorname{Res}_{b} f .
$$


Taking into account the fact that the right hand side of (16) belongs to $\mathcal{H}\left(b_{1}(M)-1\right)$ we obtain the

Corollaire 5.3. The image of $Z: \mathcal{M}(M) \longrightarrow \mathcal{H}\left(b_{1}(M)\right)$ is locally compact modulo $\mathcal{H}\left(b_{1}(M)-1\right)$.

Note that the compactness is only local because $G L(b)$ is not compact.

\section{ZETA FUnCTIONS AND ISOPERIMETRIC INEQUALITIES WITH THE LENGTH SPECTRUM}

In this section we consider a closed, orientable manifold $M$ of dimension $m$ and first Betti number $b=b_{1}(M)$. First we define a few algebraic invariants of $M$, see [3] for more detail. The characteristic map (2) induces a map between integer homology groups

$$
f_{*}: H_{m}(M, \mathbb{Z}) \longrightarrow H_{m}\left(T^{b}, \mathbb{Z}\right) \simeq \Lambda_{m} H_{1}\left(T^{b}, \mathbb{Z}\right) .
$$

Set $\alpha(M)=f_{*}([M])$ where $[M]$ is the fundamental class of $M$. The Haar measure on $H_{1}\left(T^{b}, \mathbb{R}\right)$, henceforth denoted Vol, is normalised in such a way that the lattice $H_{1}\left(T^{b}, \mathbb{Z}\right) \subset H_{1}\left(T^{b}, \mathbb{R}\right)$ has volume 1 . Take a basis $\mathbf{e}=\left\{e_{1}, \ldots, e_{b}\right\}$ de $H_{1}\left(T^{b}, \mathbb{R}\right)$, and decompose

$$
\alpha(M)=\sum_{i_{1}<\cdots<i_{m}} \alpha_{i_{1} \ldots i_{m}} e_{i_{1}} \wedge \cdots \wedge e_{i_{m}}
$$

Let us say that $\mathbf{e}$ is subordinate to $M$ if the inequality $\left|\alpha_{i_{1} \ldots i_{m}}\right| \leq 1$ holds for every coefficient of (22). Denote $E(M)$ the set of basis subordinate to $\alpha(M)$. For any basis e, let $\operatorname{Vol}(\mathbf{e})$ be the volume of the solid generated by the vectors in e. We define the algebraic volume of $M$ by setting

$$
V[M]=V(\alpha(M))=\inf _{\mathbf{e} \in E(M)} \operatorname{Vol}(\mathbf{e}) .
$$

Similarly we define the algebraic mass of $M$ by

$$
m[M]=m(\alpha(M))=\inf _{\operatorname{Vol}(\mathbf{e})=1}\left(\sum_{i_{1}<\cdots<i_{m}}\left|\alpha_{i_{1} \ldots i_{m}}\right|\right) .
$$

Remark 6.1. 1. Here is the reason for using the word "mass". Endow $H_{1}\left(T^{b}, \mathbb{R}\right)$ with a scalar product $\langle$,$\rangle and assume the Haar measure it induces coincides with Vol. Let$ $\operatorname{mass}(\alpha)$ be the usual mass norm defined on $\Lambda_{m} H_{1}\left(T^{b}, \mathbb{R}\right)$ by means of $\langle$,$\rangle (see[9]). It is$ easily proved that for all $\alpha \in \Lambda^{m} H_{1}\left(T^{b}, \mathbb{R}\right)$ we have

$$
m(\alpha)=\inf _{\langle,\rangle} \operatorname{mass}(\alpha),
$$

where $\langle$,$\rangle ranges over the set of scalar products of volume 1$.

2.The algebraic volume $V[M]$ may vanish for manifolds which are homologically essential, that is, $f_{*}([M]) \neq 0$. The invariant $V[M]$ measures the maximal non-degeneracy of $f_{*}([M])$ as a polyvector of $H_{1}\left(T^{b}, \mathbb{R}\right)$.

3. We have the following universal inequality between algebraic mass and algebraic volume (see [3] for details):

$$
\forall \alpha \in \Lambda^{m} H_{1}\left(T^{b}, \mathbb{R}\right), \quad(m(\alpha))^{b} \leq\left(\begin{array}{c}
b \\
m
\end{array}\right)^{b}(V(\alpha))^{m} .
$$


4. When $m=b_{1}$ everything boils down to the degree of the characteristic map $f$ :

$$
m[M]=V[M]=|\operatorname{deg}(f)| .
$$

Example 6.2. Let $M=M_{h}$ be an orientable surface of genus $h$. The class $\alpha\left(M_{h}\right) \in$ $H_{2}\left(T^{2 h}, \mathbb{Z}\right)$ is induced by a symplectic form with integer coefficients on $H_{1}\left(T^{2 h}\right)$ It is proved in [3] that

$$
h \geq m\left[M_{h}\right] \geq(h !)^{\frac{1}{h}} ; \quad V\left[M_{h}\right] \geq \frac{1}{(2 h-1) ! !} .
$$

6.1. The universal constant $v_{b}$. Let us consider the following variational problem from convex geometry (for more about this see [2] and -with a diffferent renormalisation- [3]). Let $B$ be a centrally symmetric convex body (CSCC) in $\left(\mathbb{R}^{b}, \operatorname{Vol}\right)$, denote $\mathcal{P}(B)$ the set of parallelepipeds which contain $B$. Define

$$
v_{\text {supp }}(B)=\frac{1}{2^{b}} \inf _{P \in \mathcal{P}(B)} \operatorname{Vol}(P) .
$$

The universal constant we are interested in is defined as

$$
v_{b}=\inf _{B} \frac{\operatorname{Vol}(B)}{v_{\text {supp }}(B)},
$$

where $B$ ranges over all CCSC's with non-empty interior.

The constant $v_{b}$ was introduced in asymptotic geometry in [2]. At the same time it appeared in different contexts, among which geometric number theory, see the introduction of [19] for more details.

The asymptotic rate of $v_{b}$ in $b$ is rather simple, see [14]. There are two positive constants $c, C$ such that

$$
c^{b} \leq \frac{v_{b}}{\omega_{b}} \leq C^{b}
$$

where $\omega_{b}$ is the volume of the unit ball in $\mathbb{R}^{b}$. The optimal values of $c$ and $C$ are not known. For practical purposes one can use $c=\frac{1}{\sqrt{e}}$ and $C=1$, see [19].

The exact value of $v_{b}$ is known only when $b=2$ or $v_{2}=3$, with the infimum achieved at affine regular hexagons [2]. An asymptotically correct lower estimate in $b$ is obtained in [19].

6.2. Isoperimetric inequality with the length spectrum. Now let us consider an orientable closed manifold $M$ of dimension $m$ with first Betti number $b_{1}=b_{1}(M)$. Let $g$ be a Riemannian metric on $M$, and let $\zeta_{s y s}(z)$ be its systolic zeta function.

Theorem 6.3. For any Riemannian metric $g$ on $M$ we have the following inequality :

$$
\left(\frac{b_{1} v_{b_{1}} V[M]}{\operatorname{Res}_{b_{1}}\left(\zeta_{s y s}(z)\right)}\right)^{m} \leq(\operatorname{vol}(M, g))^{b_{1}}
$$

Manifolds for which $\operatorname{dim}(M)=b_{1}(M)$ are a very interesting bunch. The $m$-torus is the simplest example but they may have much more complex topology, for instance the degree of the characteristic map (2) may take any integer value. Theorem 6.3 and Remark 6.1 combine to yield the 
Corollaire 6.4. Let $M$ be an orientable manifold of dimension and first Betti number m. For any Riemannian metric $g$ on $M$ we have

$$
m v_{m}|\operatorname{deg}(f)| \leq \operatorname{Res}_{b_{1}}\left(\zeta_{\text {sys }}(z)\right) \operatorname{vol}(M, g)
$$

where $f$ is the characteristic map.

It follows from Theorem 6.3, Example 6.2, and the lower estimate for $v_{2 h}$ given in [19] that

Corollaire 6.5. For any Riemmanian metric $g$ on an orientable surface $M_{h}$ of genus $h$ we have

$$
\frac{2 h \pi^{h}}{h !(2 h-1) ! !}\left(h+\frac{1}{2}\right)^{h}\left(\begin{array}{c}
h(2 h+1) \\
2 h
\end{array}\right)^{-\frac{1}{2}} \leq \operatorname{Res}_{2 h}\left(\zeta_{\text {sys }}(z)\right)\left(\operatorname{vol}\left(M_{h}, g\right)\right)^{h} .
$$

For large genera we deduce the following asymptotic estimate :

$$
\frac{\pi e}{2 h} \lesssim\left(\operatorname{Res}_{2 h}\left(\zeta_{\text {sys }}(z)\right)\right)^{\frac{1}{h}} \operatorname{vol}\left(M_{h}, g\right), \text { si } h \gg 1
$$

Proof of the Theorem. Consider the homology (or Abelian) cover $\widetilde{M}$ of $M$, that is, the cover whose transformation group is $\Gamma=H_{1}(M, \mathbb{Z}) /$ Tors, so $\widetilde{M} / \Gamma=M$. Let $\widetilde{g}$ be the lift to $\widetilde{M}$ of the metric $g$. Fix a point $q \in \widetilde{M}$ and denote

$$
V_{g}(q, t)=\left\{x \in \widetilde{M} \mid \operatorname{dist}_{\widetilde{g}}(q, x) \leq t\right\}
$$

the metric ball in $\widetilde{M}$ with center $q$ and radius $t$. It is well-known that the following limit

$$
\Omega_{H}(M, g)=\lim _{t \rightarrow \infty} \frac{\operatorname{vol}\left(V_{g}(q, t), \widetilde{g}\right)}{t^{b_{1}}}
$$

exists and does not depend on the choice of $q$. It is easily seen that

$$
\Omega_{H}(M, g)=V(g) \operatorname{vol}(M, g),
$$

where $V(g)$ is the volume of the unit ball of the stable norm on $H_{1}(M, \mathbb{R})$. The quantity $\Omega_{H}(M, g)$ is called asymptotic homology volume. In [3] a lower estimate for $\Omega_{H}(M, g)$ was sought/

Set $s=\frac{b_{1}-m}{m}$, it is easily seen that

$$
\Omega_{H}(M, g)(\operatorname{vol}(M, g))^{s}=V(g)(\operatorname{vol}(M, g))^{\frac{b_{1}}{m}}
$$

is invariant under dilatation of the metric : $g \leftrightarrow \lambda^{2} g$. It is proved in [3], Theorem 5.2, that for any metric $g$ on $M$ we have

$$
v_{b_{1}} V[M] \leq \Omega_{H}(M, g)(\operatorname{vol}(M, g))^{s} .
$$

This inequality and (23) combine to end the proof. 


\section{REFERENCES}

[1] T. M. Apostol, Introduction to Analytic Number Theory, New York, Springer-Verlag, 1976

[2] I. Babenko, Asymptotic volume of tori and the geometry of convex bodies, Mat. Zametki, vol. 44(1988), 177-190.

[3] I. Babenko, Asymptotic invariants of smooth manifolds, Russian Acad. Sci. Izv. Math. vol 41(1992), $1-38$.

[4] I. Babenko, Forte souplesse intersystolique de variétés fermées et de polyèdres, Ann. Inst. Fourier $52(2002), n^{\circ} 4,1259-1284$.

[5] I. Babenko, F. Balacheff, Sur la forme de la boule unité de la norme stable unidimensionnelle. Manuscripta Math. 119 (2006), n3, 347-358.

[6] D.Yu. Burago, Periodic Metrics, Advances in Soviet Math. vol. 9(1992), 205-210.

[7] F. Cerocchi, A. Sambusetti Quantitative bound distance theorem and Margulis' lemma for $\mathbb{Z}^{n}$ actions with applications to homology, preprint, arXiv:1412.6516vl.

[8] E. Ehrhart, Sur les polyèdres rationnels homothétiques à $n$ dimensions, C.R. Acad. Sci. Paris 254(1962), 616-618.

[9] H. Federer, Geometric measure theory, Springer, Berlin, 1969.

[10] H. Federer, Real flat chains, cochains and variational problems, Indiana Univ. Math. J. vol. 24(1974), 351-405.

[11] M. Gromov, Filling Riemannian manifolds, J. Diff. Geom. vol. 18(1983), 1-147.

[12] M. Gromov, Metric Structures for Riemannian and Non-Riemannian Spaces Birkhaüser, 2007

[13] G.H. Hardy, M. Riesz, The general theory of Dirichlet's series, Cambridge University Press, London, 1915.

[14] B.S. Kashin, Parallelepipeds of least volume that contain a convex body, Math. Zametki, vol. 45(1989), n2, pp. 134-135.

[15] D. Massart, Normes stables des surfaces, thèse de doctorat, Ecole Normale Supérieure de Lyon, 1996.

[16] D. Massart, Stable norms for surfaces : local structure of the unit ball at rational directions, Geometric And Functional Analysis, vol. 7 (1997), pp. 996-1010.

[17] D. Massart, H. Parlier On the homology length spectrum of surfaces, International Mathematics Research Notices, first published online June 14, 2016 doi:10.1093/imrn/rnw086

[18] J. Milnor, Eigenvalues of the Laplace operator on certain manifolds, Proc. Nat. Acad. Sci. USA, vol. 51(1964), n4, p. 542.

[19] A. Pelczyński, S.J. Szarek On parallelepipeds of minimal volume containing a convex symmetric body in $\mathbb{R}^{n}$, Math. Proc. Cambridge Philos. Soc. vol. 109(1991), pp.125-148.

[20] J.-P. Serre Cours d'arithmétique, Presses Universitaires de France, 1970.

[21] E. Shchepin, Greedy Sums and Dirichlet Series, preprint, arXiv:1110.5285v1.

[22] G. Tenenbaum, Introduction à la théorie analytique et probabiliste des nombres, collection SMF, 1995.

[23] E. Witt, Eine Identität zwischen Modulformen zweiten Grades, Abh. Math. Sem. Univ. Hamburg, vol.14(1941), 323-337.

Institut Montpelliérain Alexander Grothendieck, Université de Montpellier, CNRS UMR 5149, Place Eugène Batalllon, CC051, 34095 Montpellier CEDEX 5, France

E-mail address: ivan.babenko@umontpellier.fr, daniel.massart@umontpellier.fr 